\title{
Systemic sclerosis: Is there a treatment yet?
}

\author{
Carol M Black
}

Systemic sclerosis is, as yet, an untreatable disease and the choice and evaluation of any modifying treatment are extremely difficult. This is because: $(a)$ the disorder is heterogeneous and its severity and rate of progression highly variable; $(b)$ there is a tendency towards spontaneous improvement during the later stages of the disease, particularly within the more benign and numerically larger subset; $(c)$ the cause is unknown, its pathogenesis uncertain, and the relation between the vascular damage, immune dysfunction, and fibrosis speculative; $(d)$ there is a paucity of objective criteria for ascertaining improvement (or deterioration) in the disease, particularly with respect to visceral change.

This review will not seek to describe the numerous ways in which systemic sclerosis has been treated nor to comment on the vast number of vitamins, alternative medicine products, hormones, and surgical procedures that have been used (most of which have been heralded with great enthusiasm only to be abandoned after critical evaluation) but to suggest a logical approach to the use of drugs in systemic sclerosis and then to discuss some recent therapeutic ventures.

Before a treatment is decided upon for any aspect of the disease the two most important tasks for the doctor are to classify the patients and to endeavour to recognise the 'at risk' population.

Subsets of systemic sclerosis (Reproduced, with permission, from I Rheumatol 1988; 15: 202-5.')

Diffuse cutaneous systemic sclerosis*

Onset of Raynaud's phenomenon within one year of onset of Onset of Raynaud's phenomenon with
skin changes (puffy or hidebound)

skin changes (puffy or hidebound)

Presence of tendon friction rubs

Early and significant incidence of interstitial lung disease, oliguric renal failure, diffuse gastrointestinal disease, and myocardial disease

Absence of anticentromere antibodies

Nailfold capillary dilatation and capillary destruction $\dagger$

Antitopoisomerase antibodies (30\% of patients)

Limited cutaneous systemic sclerosis

Raynd's phenomenon for years (occasionally decades)

Skin disease limited to hands, face, feet, and forearms (acral) or absent

or absent
A significant late incidence of pulmonary hypertension with or

A significant late incidence of pulmonary hypertension with or
without interstitial lung disease, trigeminal neuralgia, skin without interstitial lung disease

calcifications, telangiectasia
high incidence of anticentromere antibodies (70-80\%)

A high incidence of anticentromere antibodies ( $70-80 \%)$
Dilated nailfold capillary loops, usually without capillary dropout

*Experienced observers note some patients with diffuse systemic sclerosis who do not develop organ insufficiency and suggest the term chronic diffuse systemic sclerosis for these patients.

tNailfold capillary dilatation and destruction may also be seen in patients with dermatomyositis, overlap syndromes, and undifferentiated connective tissue disease. These syndromes may be considered as part of the spectrum of scleroderma associated disorders.
An important international step in classification has been the agreement by a number of doctors, as outlined in a recent editorial, ${ }^{1}$ that there are two major variants of systemic sclerosis: the diffuse cutaneous and the limited cutaneous subtypes (table). Choice of any treatment and design of any therapeutic trial should be planned with these distinct variants taken into account; if not, the results may well be meaningless. For example, patients with late stage diffuse disease are usually not suitable for clinical trials as measurable improvement can hardly be expected when advanced fibrosis, vascular damage, and tissue atrophy are present; equally, aggressive disease modifying drugs are hardly justified in patients who have indolent disease with limited extensions and in whom measurable change will probably be absent.

Recognition of the at risk group before the disease cascade gathers momentum is equally important. Possible helpful clues are circulating antibodies, cytokine production, and nailfold changes. Such identification allows for 'protective' therapeutic intervention. These ideas should also be extended to the earliest possible diagnosis of internal organ involvement, which again might allow for its containment. For example, fine cut ( $3 \mathrm{~mm}$ ) computed tomography scans of the lungs, measurement of cytokines, growth factors and procollagen peptides in bronchoalveolar lavage fluid might, in the future, aid the recognition of lung disease, now the major cause of death in scleroderma.

No single drug or combination of drugs has proved satisfactory in the treatment of systemic sclerosis in suitably controlled prospective trials. This is perhaps understandable when one considers that concepts of systemic sclerosis are still evolving.

Obviously, development of the scleroderma lesion is complicated and entails several events that may occur simultaneously or in sequence. These almost certainly include inflammation, autoimmune attack, vascular damage, fibroblast activation by cytokines and growth factors, and interaction between different components of the extracellular matrix. There are, therefore, several different potential points of therapeutic intervention.

Although there is no good evidence for a generalised derangement of immune cell function, specific immune impairment may be important, ${ }^{23}$ and immunosuppression might play a part, especially if used at the right stage of the disorder: hypothetically such drugs could alter suggested interactions between immunocompetent cells, their cytokine products, and fibroblast function. Lack of mechanistic 
knowledge has necessitated a 'blunderbuss' approach with non-specific agents. Both alkylating agents and antimetabolites have been used. Chlorambucil studies have given mixed results. Early reports were hopeful, ${ }^{4}$ but Steigerwald, in an open study in 1985, could not substantiate these, ${ }^{5}$ and a recent three year, double blind, controlled trial was negative. ${ }^{6}$ The same historical sequence was found with 5-fluorouracil; an initial pilot study by Casas showed significant objective improvement, ${ }^{7}$ but a subsequent controlled study by the same group failed to confirm their original results. A third alkylating agent, cyclophosphamide, known to block humoral as well as certain cellular responses, has been inadequately investigated as a single agent, ${ }^{8-10}$ but these reports were not encouraging. Uncontrolled data suggest that as part of a combination treatment with steroids and plasmapheresis it may be more effective. ${ }^{11}$ The extent to which cyclophosphamide contributed to improvement with this combination is, however, unclear. Wollheim and Akesson tried to separate these effects, but as all their patients received prednisolone the results, which showed a tendency in favour of the triple theory, are not definitive. ${ }^{12}$ Removal of cytokines rather than autoantibodies, which may be markers of subsets and not pathogenic agents, is theoretically attractive. There have been several uncontrolled (or inadequately controlled) studies of the efficacy of plasma exchange, ${ }^{13-15}$ and a properly performed, randomised, controlled trial is needed.

The theoretical rationale for lymphoplasmapheresis may be sounder than plasma exchange, and a preliminary report by Weiner was encouraging. ${ }^{16}$ Considering the almost certain need to target the immune system and the nonselectivity of the immunosuppressive drugs, O'Dell et al tried total lymphoid irradiation and found it wanting with, in addition, the possible acceleration of visceral disease in the irradiated patients. ${ }^{17}$

Targeting the immune systems more specifically is, however, still an attractive idea and the use of rabbit antithymocyte-globulin is worthy of investigation. To date only two cases have been reported, ${ }^{18}$ but other studies are currently in hand.

Use of the antimetabolites 6-thioguanine and methotrexate has been reported in a few cases. ${ }^{1920}$ The information is, however, too scant to provide any useful conclusions. Azathioprine has been more widely used-always, unfortunately, in uncontrolled studies ${ }^{821}$; the authors considered the drug to be beneficial, however.

Cyclosporin A has been used in a few patients with positive results, ${ }^{22}{ }^{23}$ but high doses of the drug have been followed by reports of hypertension and renal failure, both of which might be attributed to either the drug or scleroderma. A lower dose regimen may be a sensible compromise. Such a study is in progress, ${ }^{24}$ but inadequate numbers of patients have been studied to date.

The interferons alfa and gamma are currently being evaluated in several countries. Recombinant interferon gamma has immunoregulatory activities and is also a potent inhibitor of collagen production by normal and scleroderma dermal fibroblasts in vitro. A recent pilot study by Kahan et al suggests that interferon gamma may be beneficial, ${ }^{25}$ and a blinded controlled study is indicated. Interferon alfa is a less potent inhibitor of collagen synthesis than interferon gamma but unlike interferon gamma it does not activate the class II cell surface antigens-this may be a distinct advantage in scleroderma, which is an HLA-DR linked disease. Interferon alfa is under trial in the United Kingdom, and a recent pilot study ${ }^{26}$ is encouraging enough to warrant a definitive study.

To date there is obviously no general agreement on the place of these drugs. To use them to the greatest effect more information is urgently needed on the specific immune defect so that targeting can be achieved and the drugs used at the most effective stage of the diffuse disorder-one suspects this stage to be the earliest possible beginnings. These drugs should not be used for late stage, stable diffuse or limited cutaneous disease.

Drugs which target collagen synthesis, such as colchicine and D-penicillamine, have been used in the treatment of scleroderma for many years. Neither is the answer to the fibrotic deposition, but consensus opinion is that Dpenicillamine is the most useful. The drug must be used correctly to derive the maximum benefit, and the most suitable subjects for treatment are those with widespread active skin disease. Long term treatment in a dose around $500-750 \mathrm{mg}$ is needed. Skin changes in the diffuse form of the disease are often rapid in the first six to 12 months and show a limited response to all known forms of treatment-Dpenicillamine included. The pace of change then usually slows down with regression occurring in many areas. D-Penicillamine should be continued throughout this whole period and the dose maintained until there is no further improvement in skin thickening. The drug may then be reduced, but low dose treatment should be maintained for many years-some recommend 10 years. ${ }^{27}$ The patients who present after spontaneous regression of skin changes should not be treated.

Anti-inflammatory drugs have been tried and found wanting. Steroids are potentially toxic and their use has been restricted to patients with myositis, symptomatic serositis, the oedematous phase of skin disease, and, occasionally, refractory arthritis.

Raynaud's phenomenon is a prominent feature of systemic sclerosis and may herald the development of the disease and precede it by many years. It is an aspect of the disease which may be alleviated, though the response to treatment is variable. This idiosyncratic response may reflect the stage of the disease. It is conceivable that the Raynaud's phenomenon results from endothelial damage, subintimal thickening, and consequent reduction in vessel size and that this, rather than exaggerated vasospasm, is the primary event. If this is so, the patients with structural damage will respond rather poorly to vasodilators alone. In uncomplicated cases simple measures, such as 
warm layered clothing, underwear, hats and scarves, electrically heated gloves, hand warmers, the avoidance of cold, skin care, and abstention from tobacco, may suffice. For more frequent prolonged attacks, which interfere with daily living, a vasodilator may be tried, such as nifedipine, ketanserin, thymoxamine, diltiazem, or captopril. If the attacks are accompanied by ulceration and threatened gangrene intravenous prostacyclin or prostaglandin may be needed. Secondary infection requires antibiotics and, sometimes, surgery. Frank gangrene will also require surgery in many instances. Cervical sympathectomy should be avoided, though lumbar sympathectomy still has a place.

Structural widespread vascular damage is central to the pathogenesis of scleroderma and a logical extension of this has been to seek for drugs to protect injured endothelial cells and to prevent platelet aggregation and subsequent release of platelet derived mediators. This search has been disappointing to date. Ketanserin, a serotonin antagonist, although useful in Raynaud's phenomenon, does not improve skin or visceral disease. ${ }^{28}$ Alphamethyldopa with propranolol was equally ineffective, ${ }^{29}$ and dipyridamole and aspirin, although reducing the circulating plasma concentrations of $\beta$ thromboglobulin or circulating platelet aggregates were not effective in a randomised double blind trial. ${ }^{30}$ Captopril, the angiotensin converting enzyme inhibitor, has been so successful in the treatment of renal crisis that the use of this drug or another angiotensin converting enzyme inhibitor has been considered for the primary and possibly prophylactic treatment of vascular disease.

It can be seen that there is as yet no treatment for scleroderma. A careful consideration of the heterogenicity of the disorder and its natural history, together with the subset and stage of disease of the individual patient, can maximise the use of the drugs currently available. Possibly more importantly, the level and degree of activity of research throughout the world into the cause and pathogenesis of the condition may eventually result in early rational effective treatment.

1 LeRoy E C, Black C M, Fleischmajer R, et al. Scleroderma (systemic sclerosis): classification, subsets and pathogenesis. f Rheumatol 1988; 15: 202-5.

2 Kratz L E, Bongham J A, Needleman B W. Association between $T$ cell antigen receptor gamma gene restriction, fragment length polymorphism and progressive systemi sclerosis. Arthritis Rheum 1989; 32: 534

3 Kahaleh M B, LeRoy E C. Interleukin-2 in sclerodermacorrelation of serum level with extent of skin involvement and disease duration. Ann Intern Med 1989; 110: 446-50.
4 Mackenzie A H. Prolonged alkylating drug therapy is beneficial in systemic scleroderma. Arthritis Rheum 1970; 13: 334 .

5 Steigerwald J C. Chlorambucil in the treatment of progressive systemic sclerosis. In: Black C M, Myers A R, eds. Systemic systemic sclerosis. In: Black CM, Myers A R, eds. Systemic
sclerosis (scleraderma). New York: Gower Medical, 1985: sclerosis

6 Furst D E, Clements P J, Hillis $S$, et al. Immunosuppression with chlorambucil versus placebo, for scleroderma. Results of a three year, parallel, randomized, double-blind study. Arthritis Rhewom 1989; 32: 584-93.

7 Casas J A, Subhauste C P, Alarcon G S. A new promising treatment in systemic sclerosis: 5-fluorouracil. Ann Rheum Dis 1987; 46: 763-7.

8 Saporta L, Godeau P, Delrieu F, et al. La sclérodermie géneralisée. Traitement par les immunosuppresseurs. Nouv Presse Med 1972; i: 1929-30.

9 Hurd E R, Giuliano V J. The effect of cyclophosphamide on $B$ and $T$ lymphocytes in patients with connective tissue $B$ and T lymphocytes in patients with conn
diseases. Arthritis Rheum 1975; 18: 67-75.

10 Tolchin S F, Winkelstein A, Rodnan G P, Pan S F, Nankin H R. Chromosome abnormalities from cyclophosphamide therapy in rheumatoid arthritis and progressive systemic sclerosis (scleroderma). Arthritis Rheum 1974; 17: 375-82.

11 Dau P C, Kahaleh M B, Sagebiel R W. Plasmapheresis and immunosuppressive drug therapy in scleroderma. Arthritis Rheum 1981; 24: 1128-36.

12 Wollheim F A. Akesson A. Treatment of systemic sclerosis in 1988. Semin Arthritis Rherem 1989; 18: 181-8.

13 McKune M A, Winkelmann R K, Osmundson P J, et al. A controlled study of plasmapheresis in scleroderma. $\mathcal{f}$ Clin Apheresis 1983; 1: 206-14.

14 O'Reilly M J, Talpos G, Roberts V C, White J M, Cotton $L$. Controlled trial of plasma exchange in treatment of Raynaud's syndrome. $B r \mathcal{Y M e d} 1979 ; \mathrm{i}$ : $1113-5$.

15 Capodicasa G, De Santo N G, Galione A, et al. Clinical effectiveness of apheresis in the treatment of progressive systemic sclerosis. Int $\mathcal{F}$ Artif Organs 1983; 6: 81-6.

16 Weiner S R, Kono D H, Osterman H A, Levy J, Paulus H E Pitts $W \mathbf{H}$. Preliminary report on a controlled trial of apheresis in the treatment of scleroderma [Abstract]. Arthritis Rheum 1987; 30: S27.

17 O'Dell J R, Steigerwald J C, Kennaugh R C, Hawkins R, Holers V M, Kotzin B L. Lack of clinical benefit after treatment of systemic sclerosis with total lymphoid irradiation. F Rheumatol 1989; 16: 1050-4.

18 Goronzy J J, Weyand C $M$. Therapeutic effects of $T$ lymphocyte depletion in progressive systemic sclerosis lymphocyte depletion in progressive systen

19 Demis D J, Brown C S, Crosby W H. Thioguanine in the treatment of certain autoimmune, immunologic and related diseases. Am $\mathcal{f}$ Med 1964; 37: 195-205.

20 Van den Hoogen F H J, Boerbooms A M Th, Van de Putte L B A. Methotrexate treatment in systemic sclerosis. Am $\mathcal{J}$ Med 1989; 87: 116-7.

21 Kurwa A R, Denman A M. Scleroderma treated with immunosuppressive drugs. Br f Dermatol 1979; 101: 56-7.

22 Zachariae $\mathrm{H}$, Zachariae $\mathrm{E}$. Cyclosporin $A$ in systemic sclerosis. Br $\mathcal{F}$ Dermatol 1987; 116: 741-2.

23 Appelboom T, Itzowitch D. Cyclosporine in successful control of rapidly progressive scleroderma. $A m \mathcal{F} \mathrm{Med} 1987$; 82: $866-7$.

24 Russell $M$ i $L$, Schachter $R$ A. Cyclosporin treatment of scleroderma (PSS). [Abstract]. A rihritis Rheum 1988; 31 scleroderma (
(suppl 4): S5i

25 Kahan A, Amor B, Menkes C J, Strauch G. Recombinant interferon- $\gamma$ in the treatment of systemic sclerosis. $A m \mathcal{J}$ Med 1989; 87: 273-7.

26 Stevens $W M R$, Black C M, Cohen M G, Maddison P, and the U.K. scleroderma study group multicentre pilot study of alpha interferon in recent onset diffuse cutaneous disease. Proceedings of the Medical Research Society (in press)

27 Medsger T A Jr. Treatment of systemic sclerosis. Rheumatic Diseases Clinics of North America 1989; 15: 513-31.

28 Siebold J R, Jageneau A H M. Treatment of Raynaud's phenomenon with ketanserin, a selective antagonist of the serotonin 2 (5-HT2) receptor. Arthritis Rheum 1984; 27 $139-46$.

29 Fries J F, Wasner C, Brown J, Feignbaum P. A controlledtrial of antihypertensive therapy in systemic sclerosis (scleroderma). Ann Rheum Dis 1984; 43: 407-10.

30 Beckett V L, Conn D L, Fuster V, et al. Trial of plateletinhibiting drug in scleroderma. Double-blind study with dipyridamoleand aspirin. Arthritis R heum 1984;27: 1137-43. 